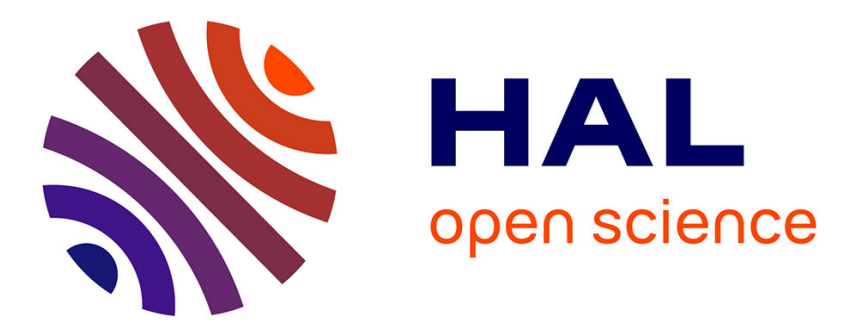

\title{
Effect of finite amplitude of bottom corrugations on Fabry-Perot resonance of water waves
}

\author{
Jie Zhang, Michel Benoit
}

\section{To cite this version:}

Jie Zhang, Michel Benoit. Effect of finite amplitude of bottom corrugations on Fabry-Perot resonance of water waves. Physical Review E , 2019, 99, pp.053109. 10.1103/PhysRevE.99.053109 . hal02558399

\section{HAL Id: hal-02558399 \\ https://hal.science/hal-02558399}

Submitted on 29 Apr 2020

HAL is a multi-disciplinary open access archive for the deposit and dissemination of scientific research documents, whether they are published or not. The documents may come from teaching and research institutions in France or abroad, or from public or private research centers.
L'archive ouverte pluridisciplinaire HAL, est destinée au dépôt et à la diffusion de documents scientifiques de niveau recherche, publiés ou non, émanant des établissements d'enseignement et de recherche français ou étrangers, des laboratoires publics ou privés. 


\title{
Effect of finite amplitude of bottom corrugations on Fabry-Perot resonance of water waves
}

\author{
Jie Zhang and Michel Benoit* \\ Aix Marseille Univ, CNRS, Centrale Marseille, IRPHE (UMR 7342), Marseille, France
}

(Received 11 February 2019; published 15 May 2019)

\begin{abstract}
Recently, the mechanism of Fabry-Perot (F-P) resonance in optics was extended to monochromatic water waves propagating in a domain with two patches of sinusoidal corrugations on an otherwise flat bottom. Assuming small-amplitude surface waves, an asymptotic linear analytical solution (ALAS) was derived by L. A. Couston et al. Phys. Rev. E 92, 043015 (2015). When resonance conditions are met, the ALAS predicts large amplification of the incident waves in the resonator area between the two patches of corrugations. Based on the ALAS, the amplitude of these standing waves is expected to increase exponentially with the relative amplitude of bottom corrugations ( $\delta=d / h$, where $d$ is the corrugation amplitude and $h$ the still water depth). In the present work, we examine the effects associated with the assumptions made in deriving the ALAS regarding the effect of a finite amplitude of bottom corrugation (i.e., finite value of $\delta$ ), still in the linear wave framework. F-P resonance is studied by means of highly accurate numerical simulations, considering either the exact linear water wave problem (system A) or an approximate problem with a first-order expansion of the bottom boundary condition (system B). The numerical model is first validated on a Bragg resonance case, through comparisons with the ALAS, experimental measurements, and existing numerical simulations, showing its ability to represent well the so-called wave-number downshift of Bragg resonance (i.e., the slight decrease in the incident wave number where maximum resonance is reached in comparison with the value predicted by the ALAS). We then analyze how this downshift affects the F-P resonance, especially when the corrugations are of finite amplitude, i.e., $\delta$ varying from 0.05 to 0.4 . The wave-number downshift appears to have a strong effect on the F-P resonance for $\delta>0.1$ : very low wave amplification manifests for the wave number predicted by the ALAS. However, when the incident wave number is slightly decreased (by an amount increasing with $\delta$ ) the F-P resonance case can be recovered, and the maximum amplification values are found to be close to the predictions from the ALAS (e.g., up to a factor of about 27 for $\delta=0.4$ ). The variations of the reflection coefficient and enhancement factor obtained from systems A and B as a function of the incident wave number are discussed and compared to ALAS predictions. In particular, it is found that the resonance peak is extremely narrow when $\delta=0.2$ and 0.4 .
\end{abstract}

DOI: 10.1103/PhysRevE.99.053109

\section{INTRODUCTION AND OBJECTIVES}

In optics, a Fabry-Perot (F-P) etalon is an interference device first described by two young French physicists at the University of Marseille (France) at the end of the 19th century [1]. Typically it consists of two parallel highly reflecting mirrors with a small interval; the incident light waves passing through will form interference fringes after a series of reflections. In the past century, the F-P resonance mechanism has been comprehensively studied and applied to different fields of physics [2,3].

In analogy to light waves, water waves can constructively interfere when propagating over a region with a constant water depth superimposed on two sets of small periodic bottom corrugations or bars. Recently, Couston et al. [4] studied water wave F-P resonance within the framework of linear potential wave theory. Based on the asymptotic linear theory, they found that significant amplification of incident regular waves with particular wave numbers can be expected over the flat-bottom area between two sets of bottom corrugations.

The mechanism behind this resonance is the well-known Bragg scattering (or resonance), which has been extensively

\footnotetext{
*benoit@irphe.univ-mrs.fr
}

studied. By applying linear perturbation method, Davies [5] and Davies and Heathershaw [6] have shown theoretically that simple harmonic waves will be scattered due to wavebottom interactions when passing through a finite number of sinusoidal rigid bars on an otherwise flat bottom. Experimental demonstrations confirming the effects of sandbars [6,7] were available soon after the theory was introduced. When the incident wavelength is twice that of the sandbars, Bragg resonance takes place. However, Davies and Heathershaw's regular perturbation method [6] fails when it is close to the resonance condition (i.e., the reflection coefficient becomes unbounded for resonance condition for a large number of bars). By considering two wave components with opposite propagation directions on the surface and introducing a cutoff frequency, Mei [8] developed an analytical approximate theory via multiple-scale perturbation method. This theory is able to predict the resonance. The leading order of Mei's theory agrees reasonably well with Heathershaw's experiments with the same linearization assumptions adopted by Davies [7]. Yu and Mei [9] also pointed out the unreliability for shore protection using Bragg resonance: it may result in suppression of waves after the sandbars, but amplification is also possible when considering reflection from the shoreline. 
Higher-order Bragg resonance can be defined by the number of components participating in the interactions. The aforementioned case is denoted class I Bragg resonance, which is second-order triad interactions (one bottom and two surface waves). In class II Bragg resonance, doubly sinusoidal corrugations on the bottom and two surface wave components interact $[10,11]$. Note that the F-P resonance, which includes two corrugation modes on the bottom, involves only second-order interactions. Such resonances are classified as $\mathrm{I}_{2}$ Bragg resonance (see [12]). In class III, monochromatic sinusoidal corrugations and three surface wave components are included [13]. The aforementioned high-order Bragg resonances are third-order quartet wave-bottom interactions. Higher-order nonlinearities of incident waves and bottom corrugations can be included by keeping more terms in the perturbation methods $[10,14]$. However, the increase in accuracy comes at the expense of simplicity of formulations.

With a family of special shapes of bottom corrugations, the constraints on the bottom steepness and/or amplitude can be released by using Floquet theory for linear wave motion [15-17]. Recently an experimental demonstration of this theory was realized [18]. However, in this case the bottom corrugations are no longer sinusoidal perturbations.

Alternatively, the nonlinearities associated with surface waves and bottom corrugations were also studied by using different numerical models solving the water wave problem to different target orders. For instance, using a boundary integral equation method, Dalrymple and Kirby [19] placed the bottom elements directly on the bars, keeping an exact bottom elevation in a linear wave framework. Kirby [20] extended the mild-slope equation of Berkhoff to the Bragg resonance case considering not only the bars on the seabed, but also the variable mean elevation of the bottom. Porter [21] extended the equation to the three-dimensional (3D) case. The high-order spectral (HOS) method developed by Dommermuth and Yue [22] was used to study the high-order Bragg resonance with the fourth-order (for both bottom and free surface) model [13].

The present work mainly focuses on a better understanding of the effects associated with the modeling of the bottom boundary condition on Bragg and F-P resonances within the linear wave theory framework, by taking advantage of an accurate and efficient numerical model. The linearized water wave problem as well as the assumptions associated with Bragg resonance, the F-P resonance condition, and the asymptotic linear analytical solution (ALAS) of the problem are recalled in Sec. II. The numerical model is introduced and validated against Bragg resonance experiments in Sec. III. Then the model is applied to study the F-P resonance in Sec. IV, considering various relative corrugation amplitudes, with most attention paid to the influence of the bottom boundary condition. The main conclusions and outlook for future work are summarized in Sec. V.

\section{PROBLEM DESCRIPTION AND MATHEMATICAL MODELING}

\section{A. Bathymetry for Bragg and Fabry-Perot cases}

Considering long-crested plane waves, the problem is formulated in a two-dimensional (2D) Cartesian coordinate system $(x, z)$, with the $x$ axis coinciding with the still water level and the $z$ axis pointing upwards. The elevation of the impermeable bottom is expressed as

$$
z=-\tilde{h}(x)=-h+\zeta(x)
$$

where $h>0$ is a constant water depth and $\zeta(x)$ describes the elevation of the bottom corrugations. These corrugations are assumed to have a sinusoidal shape over one zone (Bragg case) or two distinct zones (Fabry-Perot case) of finite length. In the latter case, these zones are labeled 1 and 2, respectively, and we assume that the bars have the same wavelength $L_{b}$ (and wave number $k_{b}=2 \pi / L_{b}$ ) and the same amplitude $d$. Each patch is composed of an integer number of bars $N_{j}$, thus covering a distance $L_{j}=N_{j} L_{b}$ between the abscissa $x_{j}^{s}$ and $x_{j}^{e}=x_{j}^{s}+N_{j} L_{b}$. The perturbation of the bottom elevation for each patch $j(j=1,2)$ thus reads

$$
\zeta(x)=d \sin \left[k_{b}\left(x-x_{j}^{s}\right)-\theta_{j}\right], \quad x \in\left[x_{j}^{s}, x_{j}^{e}\right],
$$

where $\theta_{j}$ is the phase of the corrugation patch $j$, chosen here to be either 0 or $\pi$ in order to have a continuous bottom profile. The distance between the two patches, denoted $L_{r}$, is called the resonator length. Without loss of generality, we set $x_{1}^{s}=0$ for the first patch (the second one then starting at $x_{2}^{s}=N_{1} L_{b}+L_{r}$ ). A representation of the F-P resonator configuration is shown in Fig. 1.

We consider monochromatic incident waves coming from $x=-\infty$ with amplitude $a$ and wave number $k$ in the region of uniform water depth $h$, associated with a wave period $T$ and an angular frequency $\omega=2 \pi / T$. The nondimensional parameter $\mu=k h$ is used as a measure of the relative water depth (or dispersive effects), and $k a$ measures the steepness of water waves (or nonlinear effects). The slope of the bottom corrugations is characterized by $k_{b} d$, and the nondimensional corrugation amplitude is defined as $\delta=d / h$.

\section{B. Linear mathematical modeling approaches 1. Exact linear model: System A}

The fluid is assumed inviscid and homogeneous with a constant density. The flow is assumed irrotational, so that a velocity potential $\phi$ can be introduced. The velocity field is then $\vec{u}=\nabla \phi$. The surface tension is neglected and the atmospheric pressure at the free surface is assumed uniform and constant in time (set here to 0 without loss of generality).

We further assume that surface waves are of small amplitude compared to both the wavelength and the mean water depth, i.e., $k a \ll 1$ and $a / h \ll 1$. In this case, the free surface boundary conditions can be linearized and applied at the still water level $z=0$. The governing equations for $\phi$ thus simplify to

$$
\begin{aligned}
\phi_{x x}+\phi_{z z}=0, & -\tilde{h}(x) \leqslant z \leqslant 0, \\
-\omega^{2} \phi+g \phi_{z} & =0, \quad z=0, \\
-\zeta_{x} \phi_{x}+\phi_{z} & =0, \quad z=-\tilde{h}(x),
\end{aligned}
$$

where $g$ is the acceleration due to gravity, and subscripts denote partial derivatives (e.g., $\phi_{x}=\frac{\partial \phi}{\partial x}$ ). The free surface elevation $\eta$ is obtained via $\eta(x, t)=-\phi_{t}(x, z=0, t) / g$. In this system, no assumption is made regarding the amplitude of 


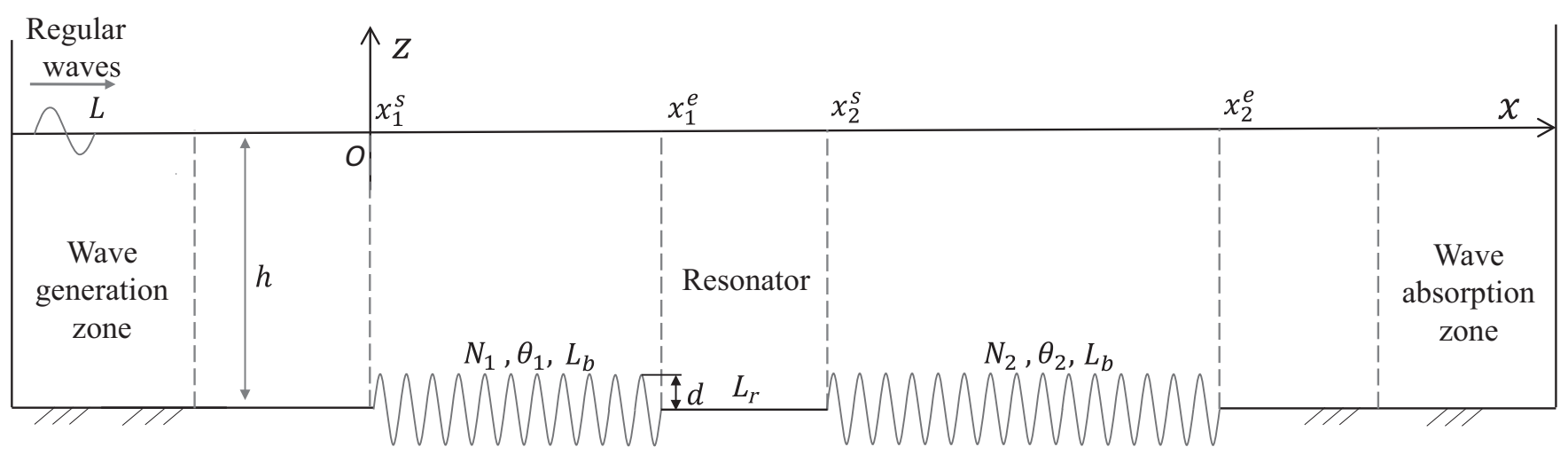

FIG. 1. Sketch of the problem setup for F-P resonance.

the bars, implying that the bottom boundary condition (BBC), Eq. (3c), is applicable for arbitrary $\delta$ and hereafter referred to as the exact BBC. System (3) is called "system A" in the following.

\section{Linear model with a small bar amplitude: System B}

As marine sandbars are usually of small amplitude with a stabilized pattern provided the sea state is steady, it is often reasonable to assume that the nonlinearity of the bars is small, i.e., $\delta \ll 1$. With this assumption, the exact BBC, Eq. (3c), can be approximated, using a truncated Taylor expansion of the potential around the mean elevation $z=-h$ and omitting second- and higher-order terms in $\delta$, as

$$
-\left(\zeta \phi_{x}\right)_{x}+\phi_{z}=0, \quad z=-h .
$$

Hereafter, Eq. (4), now applied at the uniform elevation $z=-h$, is referred to as the approximate $B B C$, and the system composed of Eqs. (3a), (3b), and (4) is called "system B." Note that with this approximate system, the Laplace equation, Eq. (3a), has to be solved over a rectangular domain of constant height $h$.

\section{Asymptotic linear model: System C}

To obtain an analytical solution of system B, Mei [8] adopted a multiple-scale expansion method and solved the leading-order problem. By introducing fast variables $(x, t)$ and slow variables $\left(x^{\prime}=\varepsilon x, t^{\prime}=\varepsilon t\right)$, assuming that $\varepsilon$ is a small parameter, the multiple-scale expansion of the velocity potential $\phi\left(x, x^{\prime}, z, t, t^{\prime}\right)$ reads

$$
\phi=\varepsilon \phi^{(1)}+\varepsilon^{2} \phi^{(2)}+O\left(\varepsilon^{3}\right),
$$

where the terms at order $O\left(\varepsilon^{3}\right)$ and higher have been neglected. Assuming that the second-order term $\phi^{(2)}$ is small compared to $\phi^{(1)}$, the first-order potential $\phi^{(1)}$ can be expressed over the $j$ th patch as

$$
\phi^{(1)}=f(z)\left[\mathcal{A}_{j}\left(x^{\prime}, t^{\prime}\right) e^{-i k x}+\mathcal{B}_{j}\left(x^{\prime}, t^{\prime}\right) e^{i k x}\right] e^{i \omega t}+\text { c.c. },
$$

where c.c. denotes the complex conjugate. $\mathcal{A}_{j}$ and $\mathcal{B}_{j}$ are the slowly varying complex amplitudes of the incident and reflected waves over patch $j$, and

$$
f(z)=-\frac{i g}{2 \omega} \frac{\cosh k(h+z)}{\cosh k h}
$$

gives the vertical dependence of the potential for the (assumed uniform) water depth $h$.

Applying Eqs. (5)-(7) to system B and enforcing the solvability and compatibility conditions (see [12] for details) for $\phi^{(2)}$, the following system is obtained, governing the evolution of amplitudes $\mathcal{A}_{j}$ and $\mathcal{B}_{j}$ over patch $j$ (hereafter referred to as "system C"),

$$
\begin{gathered}
\frac{\partial \mathcal{A}_{j}}{\partial t^{\prime}}+C_{g B} \frac{\partial \mathcal{A}_{j}}{\partial x^{\prime}}=-\Omega_{c} e^{i \theta_{j}} \mathcal{B}_{j}, \\
\frac{\partial \mathcal{B}_{j}}{\partial t^{\prime}}-C_{g B} \frac{\partial \mathcal{B}_{j}}{\partial x^{\prime}}=\Omega_{c} e^{-i \theta_{j}} \mathcal{A}_{j},
\end{gathered}
$$

where the angular frequency $\omega_{B}=\omega\left(k_{B}\right)$ and the group celerity $C_{g B}=C_{g}\left(k_{B}\right)$ of incident waves at resonant Bragg wave number $k_{B}=k_{b} / 2$ (with corresponding wavelength $L_{B}=$ $2 L_{b}$ ) are computed from linear wave theory for the water depth $h$ (note that subscript B is used for the "Bragg resonant" case as predicted by the ALAS). $\Omega_{c}$ denotes the so-called "cutoff frequency"

$$
\Omega_{c}=\frac{\omega_{B}}{4} \frac{k_{b} d}{\sinh k_{b} d} .
$$

The wave number $k$ and frequency $\omega$ of incident waves are assumed to vary in the vicinity of the Bragg resonance values, i.e., $k=k_{B}+\kappa$ with $\kappa \ll k_{B}$, and $\omega=\omega_{B}+\Omega$ with $\Omega=$ $\kappa C_{g B} \ll \omega_{B}$.

The time variation of complex amplitude can be written explicitly, e.g., $\mathcal{A}_{j}\left(x^{\prime}, t^{\prime}\right)=A_{j}\left(x^{\prime}\right) e^{i \Omega t^{\prime}}$ and $\partial \mathcal{A}_{j} / \partial t^{\prime}=i \Omega \mathcal{A}_{j}$ for the periodic steady state. Over the flat-bottom sections, the right-hand sides of Eqs. (8a) and (8b) are 0, which means that the incident and reflected waves are no longer coupled and that they propagate at the speed of their own group velocity.

One interesting feature of system $\mathrm{C}$ is that it can be solved analytically: its solution (i.e., the ALAS) is the envelopes of the amplitudes of incident and reflected waves, as functions of the slow variable $x^{\prime}$. The ALAS provides reasonable results near the resonance condition when applied to the experiments by Heathershaw [7] on Bragg resonance.

This ALAS was recently extended to the case of two patches by Couston et al. [4] to study the F-P resonance. The main results are the global reflection and transmission coefficients (for the set of two patches), which are recalled 
below:

$$
\begin{aligned}
& \left.R_{\mathrm{ALAS}}^{\mathrm{FP}}\right|_{x_{1}^{s}}=\sqrt{\frac{\left(R_{1}^{B}\right)^{2}+\left(R_{2}^{B}\right)^{2}-2 R_{1}^{B} R_{2}^{B} \cos \gamma}{1+\left(R_{1}^{B} R_{2}^{B}\right)^{2}-2 R_{1}^{B} R_{2}^{B} \cos \gamma}}, \\
& \left.T_{\mathrm{ALAS}}^{\mathrm{FP}}\right|_{x_{2}^{e}}=\sqrt{\frac{\left[1-\left(R_{1}^{B}\right)^{2}\right]\left[1-\left(R_{2}^{B}\right)^{2}\right]}{1+\left(R_{1}^{B} R_{2}^{B}\right)^{2}-2 R_{1}^{B} R_{2}^{B} \cos \gamma}},
\end{aligned}
$$

where $\quad \mathcal{R}_{j}^{B}=\mathcal{B}_{j}\left(x_{j}^{s}\right) / \mathcal{A}_{j}\left(x_{j}^{s}\right)=R_{j}^{B} \exp \left(i \alpha_{j}^{B}\right) \quad$ and $\quad \mathcal{T}_{j}^{B}=$ $\mathcal{A}_{j}\left(x_{j}^{e}\right) / \mathcal{A}_{j}\left(x_{j}^{S}\right)=T_{j}^{B} \exp \left(i \beta_{j}^{B}\right)$ are the (complex) reflection and transmission coefficients for a single patch (Bragg) case, they are functions of $\Omega / \Omega_{c}$ (see [4] for more details), evaluated at the beginning $x_{j}^{s}$ and the end $x_{j}^{e}$ of the $j$ th patch, respectively, and

$$
\gamma=\pi-2 \theta_{1}+2 k L_{r}-\alpha_{1}^{B}-\alpha_{2}^{B}
$$

In addition to Bragg resonance condition (i.e., $k=k_{B}$ ), the wave energy will be trapped within the resonator when $\gamma$ in Eq. (11) is an integer multiple of $2 \pi$, which gives a condition on the possible values of the resonator length when $k=k_{B}$ :

$$
k_{b} L_{r}^{(m)}=(2 m+1) \pi+\theta_{1}+\theta_{2}, \quad \text { with } \quad m \in \mathbb{N} .
$$

This is denoted the F-P resonance condition, under which the standing waves with the highest achievable amplitude are expected between the two patches. The nondimensional amplitude of the standing waves within the resonator is defined as the enhancement factor $E^{\mathrm{FP}}$ in [4]

$$
E_{\mathrm{ALAS}}^{\mathrm{FP}}=\frac{\mathcal{A}_{1}\left(x_{1}^{e}\right)+\mathcal{B}_{1}\left(x_{1}^{e}\right)}{\mathcal{A}_{1}\left(x_{1}^{s}\right)}=\left(1+R_{2}^{B}\right) \frac{T_{\mathrm{ALAS}}^{\mathrm{FP}}}{T_{2}^{B}} .
$$

The aforementioned results indicate that large amplification of the incident waves could take place in the resonator area, with enhancement factors greater than 2 or even larger depending on the incident wave conditions and bottom characteristics. For instance, in the case simulated in [4], the following setup is chosen: $N_{1}=11, N_{2}=15, \theta_{1}=\theta_{2}=0$, $k_{b} h=1.64, k_{b} d=0.164$ (i.e., $\delta=0.1$ ), and $k_{b} L_{r}=11 \pi$ [i.e., $m=5$ in the F-P resonance condition, Eq. (12)].

Considering each patch individually, with the Bragg condition satisfied, the reflection and transmission coefficients are $R_{1}^{B} \approx 0.597$ and $T_{1}^{B} \approx 0.803$ for the first patch and $R_{2}^{B} \approx$ 0.734 and $T_{2}^{B} \approx 0.679$ for the second patch. Relatively strong reflection of incident waves is expected, based on the principle of energy conservation, and waves passing through a single patch are of smaller amplitude. In the F-P case with precisely chosen resonator length $k_{b} L_{r}=11 \pi$, the overall reflection and transmission coefficients are $R_{\mathrm{ALAS}}^{\mathrm{FP}} \approx 0.245$ and $T_{\mathrm{ALAS}}^{\mathrm{FP}} \approx$ 0.970 . The standing waves in the resonator are amplified by a factor of $E_{\mathrm{ALAS}}^{\mathrm{FP}} \approx 2.476$.

In Fig. 2, we show the relationship between $E_{\mathrm{ALAS}}^{\mathrm{FP}}$ and $\delta$ (keeping $N_{1}=11, N_{2}=15, \theta_{1}=\theta_{2}=0, k_{b} h=1.64$, and $k_{b} L_{r}=11 \pi$ fixed). It can be observed that within the linear framework, $E_{\mathrm{ALAS}}^{\mathrm{FP}}$ increases exponentially as the amplitude of bars increases. As $\delta$ becomes larger, one can anticipate that the results from the ALAS will become unrealistic, mainly for two reasons: on one hand, the standing waves will be of a high amplitude so that nonlinear effects due to the finite wave

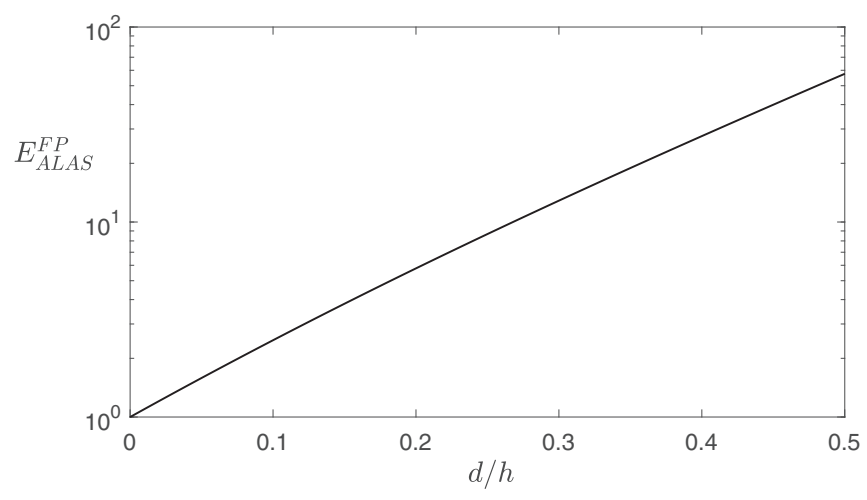

FIG. 2. Enhancement factor $E_{\mathrm{ALAS}}^{\mathrm{FP}}$ as a function of the nondimensional corrugation amplitude $\delta=d / h$. The water depth, setup of two patches, and length of the resonator are the same as those used in [4].

amplitude will be significant; on the other hand, these waves might become too steep to preserve their shape and wave breaking should occur. Regarding the ALAS, the assumption on the smallness of the bottom slope is violated, and so is the assumption of linearity of the water waves over the resonator area.

\section{NUMERICAL MODELING AND VALIDATION IN THE BRAGG RESONANCE CASE}

\section{A. Description of the numerical model}

The numerical simulations of systems A and B are performed with a highly accurate code, called WHISPERS-3D. This code is developed to solve the fully nonlinear potential wave problem with variable bottom conditions, in the form of two coupled nonlinear equations, corresponding to the two nonlinear free surface boundary conditions (FSBCs). In dimensional form for the case of a single horizontal dimension [23], they are expressed as

$$
\begin{aligned}
\eta_{t} & =-\eta_{x} \tilde{\Phi}_{x}+\tilde{w}\left[1+\left(\eta_{x}\right)^{2}\right], \\
\tilde{\Phi}_{t} & =-g \eta-\frac{1}{2}\left(\tilde{\Phi}_{x}\right)^{2}+\frac{1}{2} \tilde{w}^{2}\left[1+\left(\eta_{x}\right)^{2}\right],
\end{aligned}
$$

where $\tilde{\Phi}(x, t) \equiv \Phi(x, z=\eta(x, t), t)$ is the free surface velocity potential and $\tilde{w}(x, t) \equiv \Phi_{z}(x, z=\eta(x, t), t)$ is the vertical velocity at the free surface. Note that these equations involve only free surface variables, though a $2 \mathrm{D}$ vertical $(x, z)$ situation is modeled.

In order to march Eqs. (14) in time, the vertical velocity $\tilde{w}(x, t)$ has to be determined as a function of $[\eta(x, t), \tilde{\Phi}(x, t)]$, corresponding to a so-called Dirichlet-to-Neumann problem. The modeling approach used is presented in previous works $[24,25]$ and summarized hereafter. Following Tian and Sato [26], a spectral approach is used in the vertical to approximate the velocity potential. Using the set of orthogonal Chebyshev polynomials of the first kind, denoted $T_{n}(s), n=$ $0,1, \ldots, N_{T}$, with $s \in[-1,1]$, as an expansion basis, the potential is approximated at any given time $t$ (omitted for 
brevity hereafter) as

$$
\Phi(x, z)=\varphi(x, s) \approx \sum_{n=0}^{N_{T}} a_{n}(x) T_{n}(s),
$$

where $s(x, z, t)$ is the scaled vertical coordinate allowing us to map the water column $z \in[-\tilde{h}(x), \eta(x, t)]$ into the fixed range $s \in[-1,1]$, and the $a_{n}$ coefficients $\left(n=0,1, \ldots, N_{T}\right)$ depend upon the local abscissa $x$ (and time $t$ ).

The main steps involved in solving the Dirichlet-toNeumann problem and integrating Eqs. (14) in time are summarized as follows: (i) first, the system of governing equations composed of the Laplace equation, a Dirichlet FSBC on the potential, and the $\mathrm{BBC}$ is expressed in the $(x, s)$ coordinate system; (ii) then the approximation given in Eq. (15) is inserted into these equations; (iii) the so-called Chebyshevtau method, a variant of the Galerkin method, is used to project the Laplace equation onto the $T_{n}$ polynomials for $n=$ $0,1, \ldots, N_{T}-2$, eliminating the $s$ coordinate and giving a set of $N_{T}-1$ equations on the $a_{n}$ coefficients at each location $x$; (iv) two additional equations are obtained by considering the Dirichlet FSBC and the BBC so that a system of $N_{T}+1$ linear equations with $N_{T}+1$ unknowns $\left(a_{n}, n=0, \ldots, N_{T}\right)$ at each abscissa is formed; and (v) once this linear system is solved for the $a_{n}$ coefficients, the vertical velocity at the free surface is obtained as

$$
\tilde{w}(x, t)=\frac{2}{\tilde{h}(x)+\eta(x, t)} \sum_{n=1}^{N_{T}} a_{n}(x, t) n^{2},
$$

allowing Eqs. (14) to be integrated in time.

In WHISPERS-3D, horizontal derivatives are approximated using fourth-order finite-difference formulas using stencils of five nodes on a regular grid and an explicit third-order Runge-Rutta scheme (SSP-RK3) is used for time marching. The maximum order $N_{T}$ of polynomials in Eq. (15) determines the accuracy of the model. With this representation of the potential, the model exhibits a geometric convergence as a function of $N_{T}$, so that a high accuracy of the vertical structure of the flow can be obtained with a limited number of terms, usually in the range $N_{T} \in[5,10]$. This property was carefully verified for a number of cases with regular or irregular waves over flat or variable bottom conditions [24,25].

The linearized version of the numerical model, solving the system of Eqs. (3), was extensively studied in [27], for both flat and variable bottom profiles. The dispersion relation of the linear model was derived analytically [27],

$$
\frac{C_{N_{T}}^{2}}{g h}=\frac{1+\sum_{p=1}^{N_{T}-2} \alpha_{p} \mu^{2 p}}{1+\sum_{p=1}^{N_{T}-1} \beta_{p} \mu^{2 p}}
$$

where $C_{N_{T}}$ denotes the approximation of the phase celerity of the waves given by the model at order $N_{T}$. The computational method for obtaining the analytical expressions of $\alpha_{p}$ and $\beta_{p}$ coefficients can be found in [27].

In order to illustrate the resolving capability of the model for the water depth conditions considered here $(\mu=$ $k h \approx k_{B} h=0.82$ ), the evolution of the relative error $\mid C_{N_{T}}-$ $C_{\text {Airy }} \mid / C_{\text {Airy }}$ on the phase celerity of the linearized version of the model with respect to the exact Airy phase celerity under flat-bottom conditions [given by $\left.C_{\text {Airy }}^{2} /(g h)=\tanh (\mu) / \mu\right)$ ] is

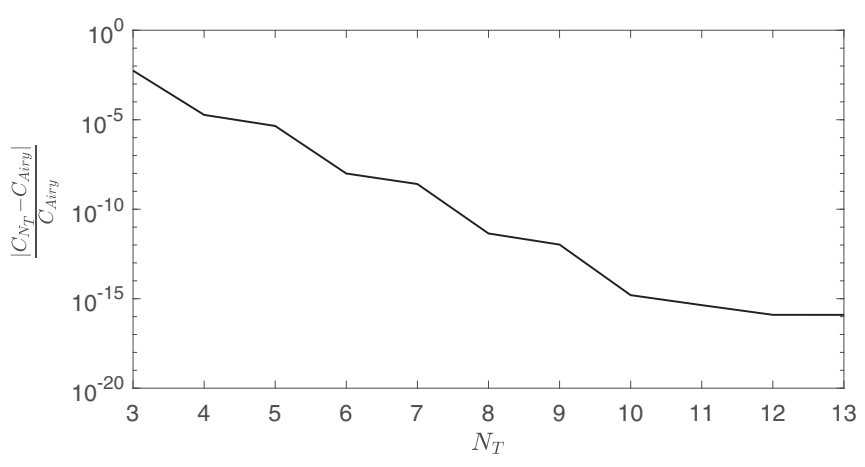

FIG. 3. Relative error on the phase celerity of the linear version of the numerical model (with respect to the exact Airy celerity) for the relative water depth $\mu=0.82$ as a function of the maximum order of polynomials $N_{T}$.

plotted as a function of $N_{T}$ in Fig. 3. It is shown that this error decreases exponentially with increasing $N_{T}$. For this relative water depth $\mu=0.82$, the difference between $C_{N_{T}}$ and $C_{\text {Airy }}$ drops below the machine precision as $N_{T}$ exceeds 12 . In the simulations performed hereafter, a value of $N_{T}=7$ will be systematically used. With this value the relative error on phase celerity is about $2 \times 10^{-9}$.

We point out that Liu and Yue [13] simulated Bragg resonance cases by solving Eqs. (14) with the (nonlinear) HOS method. In the HOS method [13,22], assuming periodic boundary conditions in the horizontal direction, the velocity potential is represented by a large number of free wave modes whose amplitudes are determined via a pseudospectral method. The problem is solved by combining a perturbation expansion method for the potential and Taylor expansions of the nonlinear FSBCs and the BBC around their mean levels, giving an approximate solution at a given target order in wave steepness. The numerical model adopted here does not assume spatial periodicity of the spatial domain, the BBC is applied at the exact position of the bottom (in system A), and the FSBCs are applied at the exact position of the free surface (in the nonlinear version of the model). The vertical variation of the potential at each horizontal node is instead represented by a series of Chebyshev polynomials given by Eq. (15). By increasing the order $N_{T}$ of this vertical approximation (and concurrently decreasing the spatial grid size), extremely accurate representations of the potential can be reached over the fluid domain, for both the linearized and the fully nonlinear versions of the code.

\section{B. Simulation results of the Bragg resonance case}

The experiments conducted by Davies and Heathershaw [6] on Bragg resonance have been intensively studied since their publication, e.g., in $[8,13,19,20,28]$. We start by simulating one case of these experiments in order to validate the linearized version of WHISPERS-3D.

In the experiments in [6], patches with $N=2,4$, and 10 bars were tested. We select the case with the longest patch $(N=10)$ because in this case stronger wave-bottom interaction is expected, resulting in significant reflection of incident waves. Besides, this case clearly showed a wave-number downshift effect and is thus considered most challenging for 


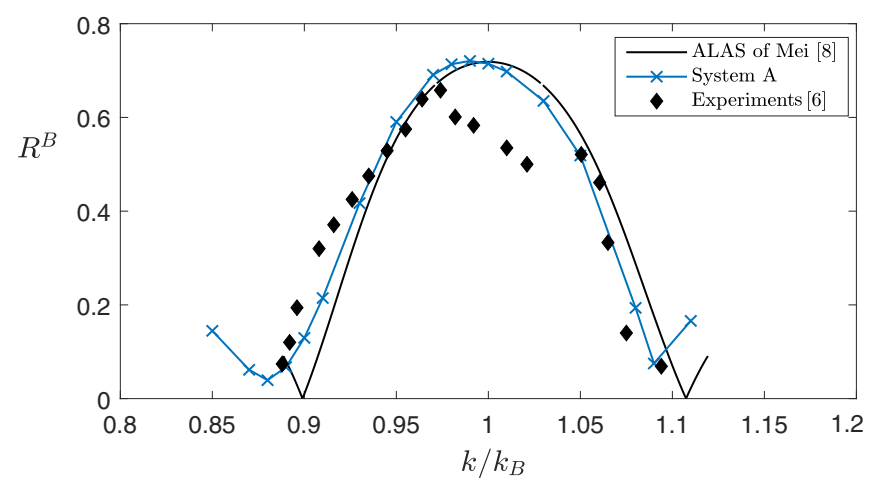

FIG. 4. The simulation results (system A) of Davies and Heathershaw's experiments [6] with 10 bars are compared with the experimental measurements. The ALAS prediction based on Mei's theory [8] is also given as a reference.

the numerical model. In the experiments, the bottom corrugations are fixed at $d=0.05 \mathrm{~m}$ and $L_{b}=1 \mathrm{~m}$. The relative corrugation amplitude $\delta=0.16$ is achieved by adjusting the water depth $h$. The wavelength of incident waves is determined from the Bragg resonance condition $L=L_{B}$. Regarding the numerical model, sinusoidal waves are generated and absorbed by using relaxation zones with $4 L_{B}$ in length. Before and after the patch of corrugations, two zones with constant water depth $h$ and length $3 L_{B}$ are used. The domain is meshed with a regular grid defined by $\Delta x=L_{B} / 64$. The time step is chosen as $\Delta t=T / 64$, giving a Courant-Friedrichs-Lewy number $\mathrm{CFL}=1(\mathrm{CFL}=C \Delta t / \Delta x$, with phase velocity $C=$ $L / T$ ). It should be noted that in the numerical simulations, instead of using the dispersion relationship of Airy wave theory, the wave period $T$ is determined by the analytical dispersion relationship, Eq. (17), of WHISPERS-3D at order $N_{T}$.

A series of runs is performed by varying the wave number of incident waves around the value $k_{B}$ corresponding to expected resonance based on the ALAS. Once a periodic state is reached over the domain, the reflected and transmitted waves are separated by Goda and Suzuki's method [29], and the corresponding reflection coefficient from the ALAS $R^{B}$ is evaluated following Eq. (6a) in [4]. The reflection coefficients are compared in Fig. 4, from which it can be concluded that the numerical results show good agreement with the experimental results as well as theoretical predictions for the primary resonance tongue.

However, it is also clear that the maximum reflection coefficient is not obtained for the Bragg condition $k_{B}=k_{b} / 2$, as is predicted by the ALAS, but for a slightly smaller wave number. This effect corresponds to the so-called wave-number downshift (or, equivalently, frequency downshift). Liu and Yue [13] explained this slight detuning by showing that the spatially averaged local wave number over the patch is always larger than the incident wave number, while it was considered uniform over the whole domain during the derivation of the ALAS. In other words, the "effective" wave number over the patch (whose mean water depth is $h$ ) is greater than the wave number of a uniform water depth $h$ (this is further discussed in Sec. IV C below). A slightly smaller incident wave number is thus required to compensate this increase due to the presence of bars; then the Bragg condition is met again.
We also note that Liu and Yue [13] obtained results very similar to those for system A in Fig. 4 using the HOS method (see Fig. 6 in [13]), although the nonlinearity of water waves is excluded in the present model. This implies that the wavenumber downshift in this configuration is mainly a BBC effect, and not a nonlinear effect. Overall, the good agreement of the present results of system A with the experimental, the ALAS, and the numerical results of [13] validates the current numerical model for the Bragg resonance case.

\section{SIMULATION AND ANALYSIS OF FABRY-PEROT RESONANCE}

\section{A. Description of the numerical setup}

Now consider the F-P resonance; we aim at investigating whether large enhancement factors (see Fig. 2) for finiteamplitude bars can be realized within the exact linear framework (system A). In other words, we focus on the effects associated with the assumption of smallness of the corrugation amplitude. To this end, the assumption of small-amplitude surface waves is preserved, and the height of standing waves in the resonator should in principle remain small for the linear approach to apply.

Regarding the numerical setup, we again follow the work in [4] as introduced in Sec. II except for the amplitude of corrugations. Here, four kinds of tests with different corrugation amplitudes are studied: $\delta=0.05,0.1,0.2$, and 0.4 . Note that, for this bottom configuration, ALAS predicts $E_{\mathrm{ALAS}}^{\mathrm{FP}} \approx 27.579$ when $\delta=0.4$. With such large amplification, even small to moderate incident waves could lead to very large standing waves, with possible dramatic effects on the local structures. For the numerical settings, the generation and absorption zones are $4 L_{B}$ in length. The domain is uniformly meshed with $\Delta x=L_{B} / 128$. The time step is chosen as $\Delta t=T / 256$, giving $\mathrm{CFL}=0.5$. The duration of the simulations depends on the corrugation amplitude $\delta$. Indeed, for F-P resonance, runs with higher bar amplitudes take more time to reach a time-periodic steady state.

\section{B. Simulations with ALAS-tuned incident waves for small to moderate corrugation amplitudes}

For the considered setup, the free surface motion consists of left- and right-propagating components with the same frequency, i.e., reflected and transmitted waves. The reflected waves are the comprehensive results of all the bars downstream at any given $x$. This suggests that no reflected wave is expected after the second patch. Standing waves are expected over the flat-bottom zones before the first patch and within the resonator. Over the patches, not only the phase of the free surface envelope but also its amplitude is slowly modulated because of the change in the total number of downstream bars. By excluding the fast oscillations, the ALAS describes the envelope of the free surface elevation and indicates the slow space modulation of the wave amplitude. Its computation formula can be found in the Appendix in [4].

In this section, we present results of simulations of system A and system B (done with the linearized version of WHISPERS-3D) using an incident wave number as predicted by 


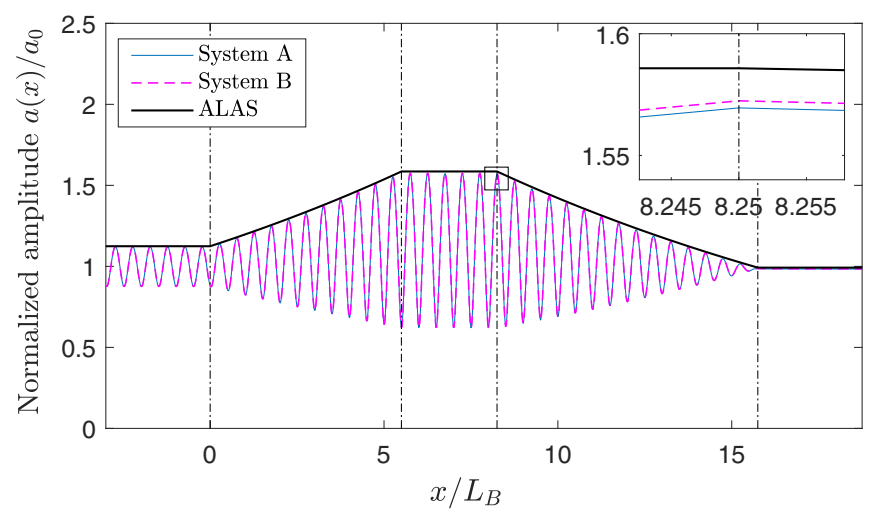

FIG. 5. Computed envelope of the free surface elevation at the end of the simulations of systems A and B $(t=100 T)$ for the case $\delta=0.05$ with the wave number $k=k_{B}$, compared to the envelope from the ALAS.

the ALAS, namely, $k=k_{B}$, for different cases with increasing values of $\delta$.

For the case with small bottom corrugation amplitude $\delta=$ 0.05 , Fig. 5 shows that the simulated results of systems A and B are almost superimposed. For this case, the reflection and enhancement coefficients predicted by the ALAS are both small, namely, $R_{\mathrm{ALAS}}^{\mathrm{FP}} \approx 0.124$ and $E_{\mathrm{ALAS}}^{\mathrm{FP}} \approx 1.586$. Nice agreement with the ALAS is observed throughout the computational domain, and the time required to achieve a quasi-steady state is less than 100 incident wave periods. This case validates the applicability of the current numerical model to the F-P resonance case.

The simulation results with the higher corrugation amplitude $\delta=0.1$ are shown in Fig. 6. The duration of simulation is now $200 T$. For this case, the results of systems A and B are still too close to be distinguished on the global scale, which means that the assumption on the BBC adopted by Davies [5] and Mei [8] remains appropriate. However, simulation results deviate slightly from the ALAS results. The reflection coefficient from system $\mathrm{A}, R_{A}^{\mathrm{FP}} \approx 0.329\left(R_{B}^{\mathrm{FP}} \approx 0.336\right.$ from system $\mathrm{B})$, is larger than the $R_{\mathrm{ALAS}}^{\mathrm{FP}} \approx 0.245$ from the ALAS, and the enhancement factor from system $\mathrm{A}, E_{A}^{\mathrm{FP}} \approx 2.385\left(E_{B}^{\mathrm{FP}} \approx\right.$ $2.397)$, is smaller than the expected $E_{\mathrm{ALAS}}^{\mathrm{FP}} \approx 2.476$ from the ALAS.

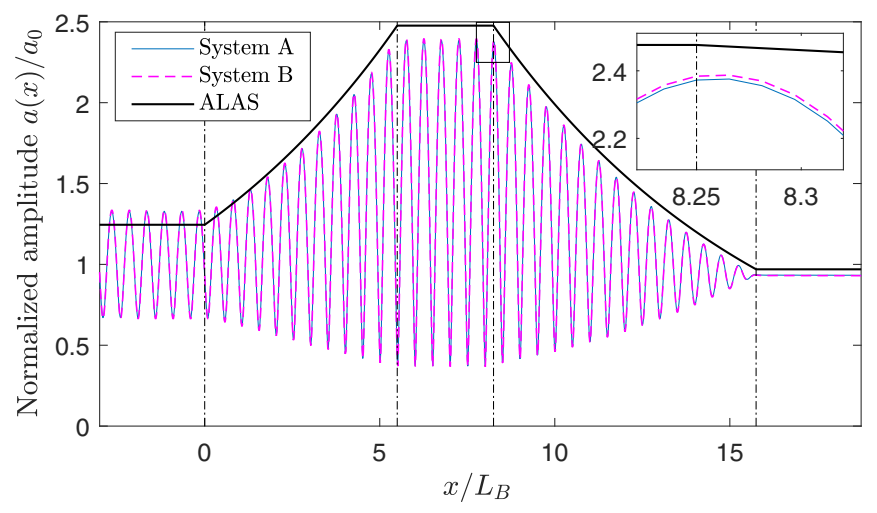

FIG. 6. Same as Fig. 5, for the case $\delta=0.1$. The simulation duration is $t=200 T$.

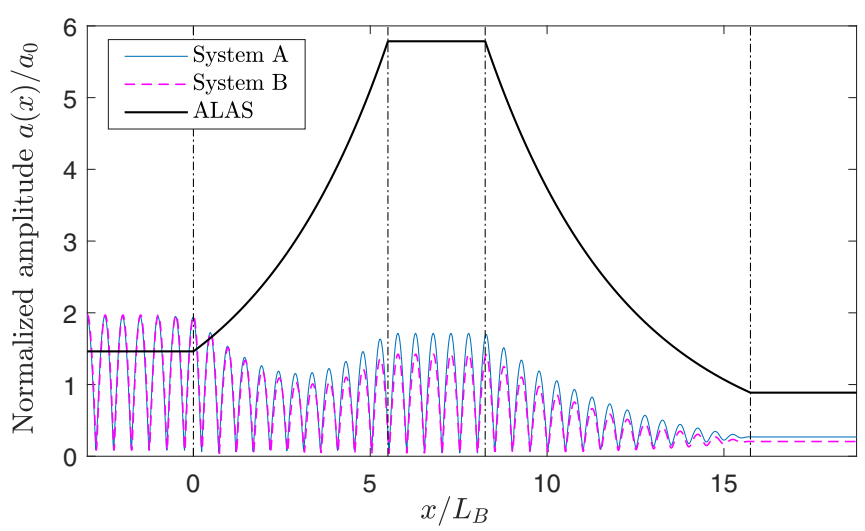

FIG. 7. Same as Fig. 5, for the case $\delta=0.2$. The simulation duration is $t=900 T$.

The corrugation amplitude is then increased to $\delta=0.2$. It is clearly shown in Fig. 7 that the results from systems $\mathrm{A}$ and $\mathrm{B}$ and from ALAS predictions are different. Before the first patch, simulations with systems A and B show that the incident waves are nearly fully reflected with a reflection coefficient $R_{A}^{\mathrm{FP}} \approx 0.956$ for system $\mathrm{A}$, resulting in little energy being transferred through the second patch of corrugations. Within the resonator, the enhancement factor does not reach the predicted value by the ALAS, $E_{\mathrm{ALAS}}^{\mathrm{FP}} \approx 5.785$ : only $E_{A}^{\mathrm{FP}} \approx$ 1.711 is obtained with system A. Clearly, the F-P resonance does not manifest in this case with either system A or system B. In addition, it is also noteworthy that the results of systems $A$ and $B$ are no longer superimposed, implying that the assumption on the smallness of bottom corrugations is less acceptable for $\delta=0.2$ or larger. Finally, we point out that this case is much more time-consuming compared to the cases with smaller corrugation amplitudes: no less than $900 T$ is required to approach the steady state.

\section{Simulations with slightly detuned incident waves for a finite corrugation amplitude}

As the linearized version of WHISPERS-3D with the exact $\mathrm{BBC}$ has been proven valid for the Bragg and F-P resonances (at least for small-amplitude bars), it is interesting to investigate whether large enhancement factors could be achieved in the case where the bottom corrugations are of finite amplitude and to analyze why the F-P resonance could not be realized for $\delta=0.2$ in the previous subsection. For this purpose, tests with finite corrugation amplitudes are performed here, namely, $\delta=$ 0.2 and then 0.4 .

\section{Slightly detuned simulations with $\delta=0.2$}

The case $\delta=0.2$ is repeated here, but now the wave number of incident waves is detuned by a small value, so that $k / k_{B}$ varies in the range $[0.92,1.10]$. The numerical parameters and settings for systems A and B remain unchanged. The effect of detuning the wave number $k / k_{B}$ on the reflection coefficient $R^{\mathrm{FP}}$ is plotted in Fig. 8. The ALAS curve is symmetric with respect to the resonance condition $k / k_{B}=1$, where a minimum value is reached. However, when departing from this value the reflection coefficient increases very rapidly, meaning 


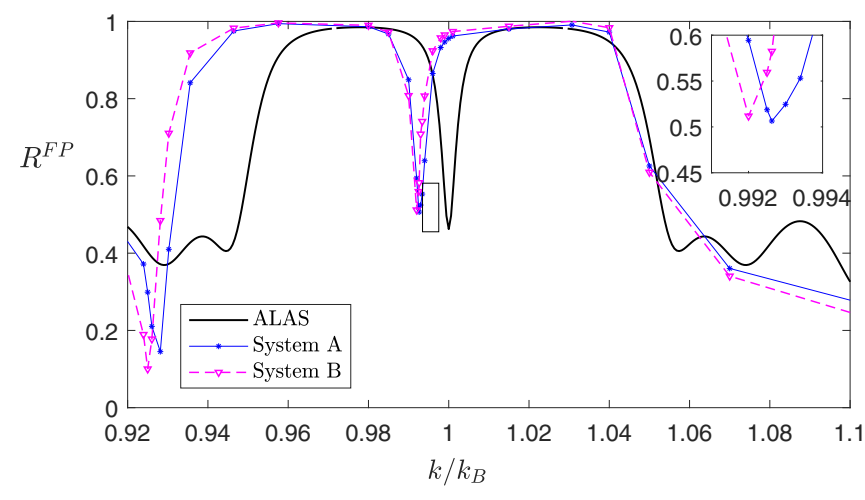

FIG. 8. Effect of the detuned wave number (normalized by $k_{B}$ ) on the reflection coefficient $R^{\mathrm{FP}}$ for systems $\mathrm{A}$ and $\mathrm{B}$. The ALAS prediction based on Eq. (10a) is also superimposed.

that the F-P resonance is sensitive to the wave number (or, equivalently, the frequency) of incident waves, especially for finite-amplitude bottom corrugations. Regarding the results for systems $\mathrm{A}$ and $\mathrm{B}$, there are clear shifts of the symmetry axis from $k / k_{B}=1$ toward smaller values. For this value of $\delta=0.2$, the difference between the exact BBC (system A) and the approximate BBC (system B) remains limited.

For the same set of simulations, the enhancement factor in the resonator is plotted in Fig. 9. Again the detuning effect is clearly visible, with a downshift of the peak of maximum $E_{A}^{\mathrm{FP}}$ towards a lower value, namely, $k \approx 0.99263 k_{B}$, for system $\mathrm{A}$.

It is verified here that $E_{A}^{\mathrm{FP}}$ is quite low for $k=k_{B}$, as shown in Fig. 7. This can be explained by the sensitivity of the F-P resonance to the incident wave number and by the wave-number downshift for finite corrugation amplitude. Following Liu and Yue [13] for Bragg resonance, we can evaluate a "mean" wave number $\bar{k}_{j}$ over patch $j$ by a numerical averaging method,

$$
\bar{k}_{j}=\frac{1}{x_{j}^{e}-x_{j}^{s}} \int_{x_{j}^{s}}^{x_{j}^{e}} k_{j}(x) d x,
$$

where $k_{j}(x)$ denotes the local wave number over patch $j$ as computed from the dispersion relation for the actual water depth $\tilde{h}(x)=h-\zeta(x)$. The ratio $\mathcal{D}_{j}=\bar{k}_{j} / k(h)$ is a measure

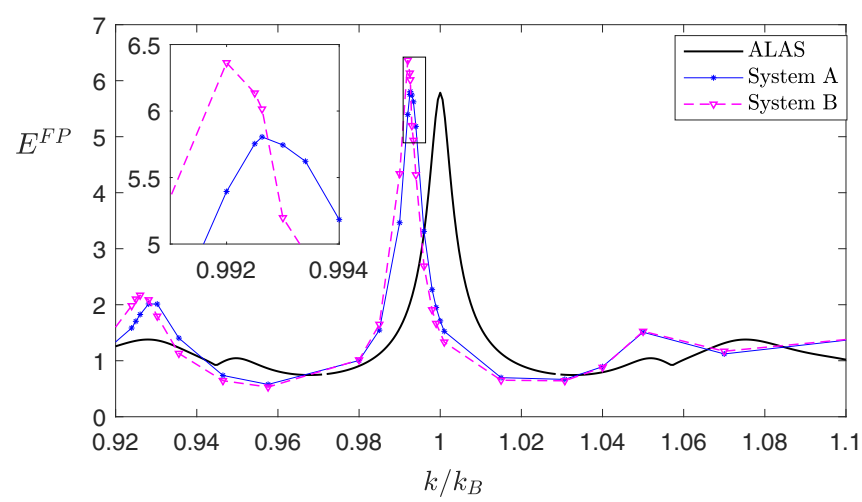

FIG. 9. Effect of the detuned wave number (normalized by $k_{B}$ ) on the enhancement factor $E^{\mathrm{FP}}$ for systems $\mathrm{A}$ and B. The ALAS prediction based on Eq. (10a) is also superimposed.

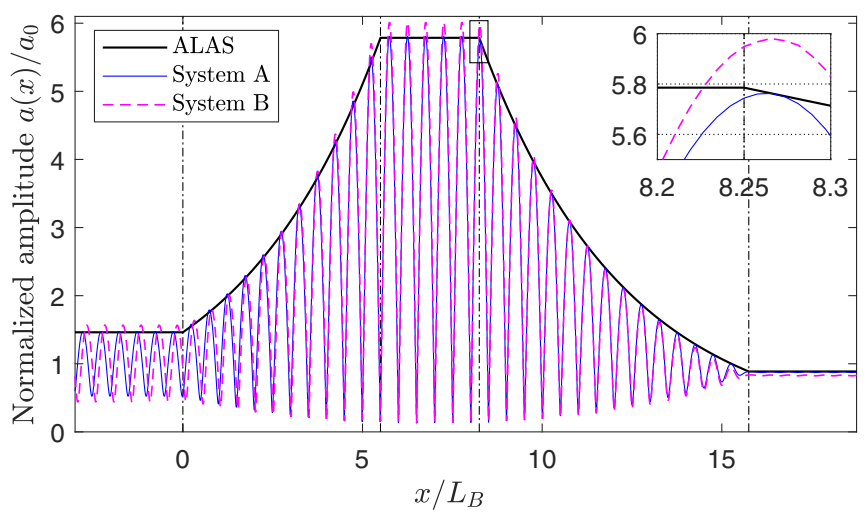

FIG. 10. Computed envelope of the free surface elevation at the end of the simulations of systems A and B $(t=900 T)$ for the case $\delta=0.2$ with the wave number slightly smaller $\left(k^{\prime}=0.99263 k_{B}\right)$ than the F-P condition, compared to the ALAS envelope (calculated for $k=k_{B}$ ).

of the effect of the undulated bottom. For the present case (same corrugation amplitude for the two patches), we obtain $\mathcal{D}_{1}=\mathcal{D}_{2} \approx 1 / 0.9933$. Based on this result, it is anticipated that the nondimensional incident wave number should be $k / k_{B}=\mathcal{D}_{1}^{-1}=0.9933$ to recover F-P resonance, which indeed is located very close to the shifted resonance condition in Figs. 8 and 9. As shown in these figures, it is speculated that the "real" resonance condition for the incident wave number in the present case falls between 0.99263 and 0.993 .

In Fig. 10, the simulation results for systems A and B using the slightly detuned incident wave number $k / k_{B}=0.99263$ are plotted, together with the ALAS envelope curve (for $k=k_{B}$ ). Good agreement is achieved between the simulated results (in particular, with system A) and the ALAS prediction regarding the maximum wave amplitude over the whole domain. It should be noted that the differences in the results for system A vs system B are due to the difference in the degree of downshift associated with each of the two systems (clearly shown in the inset in Fig. 9). Thus, the wave-number downshift is mainly a leading-order phenomenon but is also influenced by the order of approximation of the BBC.

\section{Slightly detuned simulations with $\delta=0.4$}

To demonstrate the significance of the wave-number downshift and to show that the F-P resonance can be realized even for high bottom corrugation amplitudes, an additional test for $\delta=0.4$ is performed and analyzed, with system A only. As shown in Fig. 2, the enhancement factor predicted by the ALAS for this case should be $E_{\mathrm{ALAS}}^{\mathrm{FP}} \approx 27.579$, associated with the reflection coefficient $R_{\mathrm{ALAS}}^{\mathrm{FP}} \approx 0.762$. The assumption on the smallness of bottom corrugations is obviously no longer fulfilled, and it is thus interesting to compare the results for system A (with the exact BBC) and for the ALAS.

Considering that the "exact" condition for F-P resonance is unknown, Eq. (18) is used to provide the first guess of the shifted F-P resonance condition, leading to $k=0.972 k_{B}$. Then the proper incident wave number associated with the largest enhancement factor $E_{A}^{\mathrm{FP}}$ is found by exploring a range of wave numbers in the vicinity of this value. The result 


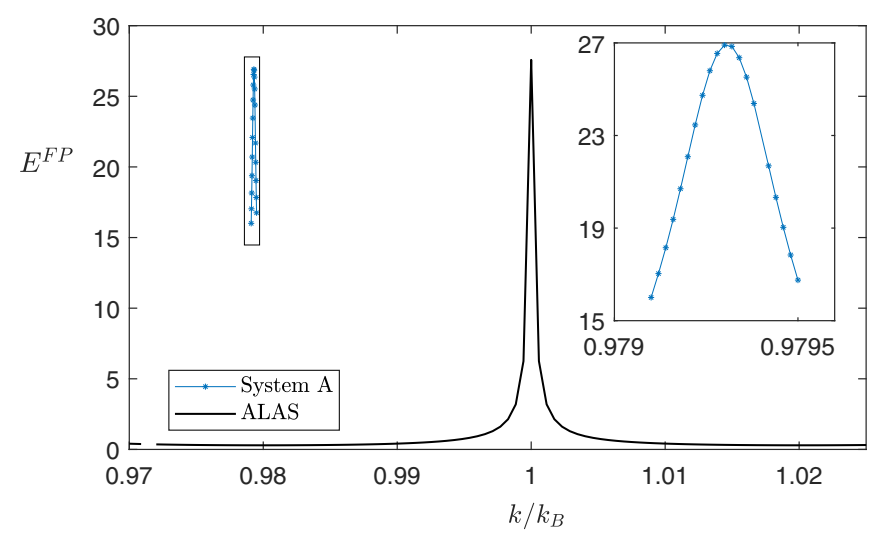

FIG. 11. Effect of the detuned wave number (normalized by $k_{B}$ ) on the enhancement factor $E^{\mathrm{FP}}$ for system $\mathrm{A}$. The ALAS prediction based on Eq. (10a) is also superimposed.

regarding the enhancement factor is shown in Fig. 11. The region where the F-P resonance takes place is extremely sharp and narrow, meaning that the resonance condition is very strict. Among the set of discrete values considered in the simulations, the detuned incident wave number corresponding to the maximum enhancement factor is $k=0.979313 k_{B}$. The "exact" resonance condition is found to lie between $0.9793 k_{B}$ and $0.979317 k_{B}$. It should be noted that even though the amplitude of bottom corrugations is high, the prediction of the value of the maximum $E^{\mathrm{FP}}$ by the ALAS is quite reliable: system $\mathrm{A}$ gives $E_{A}^{\mathrm{FP}} \approx 26.918$ at maximum, which is quite close to $E_{\mathrm{ALAS}}^{\mathrm{FP}}=27.579$. Such a close match was not guaranteed a priori, as the wave number corresponding to the maximum enhancement factor is significantly shifted downwards.

As a confirmation of the realization of F-P resonance, the envelope of the simulation done with system A using the incident wave number $k=0.979313 k_{B}$, corresponding to the maximum enhancement factor in Fig. 11, is shown in Fig. 12. A reasonable agreement is found, confirming the possibility of reaching large enhancement factors in the resonator within

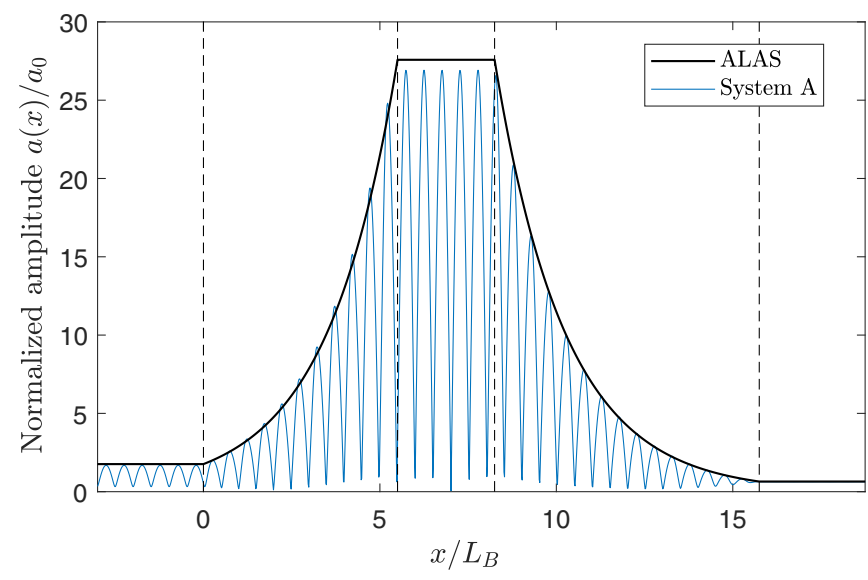

FIG. 12. Computed envelope of the free surface elevation at the end of the simulations with system A $(t=10000 T)$ for the case $\delta=$ 0.4 with the wave number slightly smaller $\left(k^{\prime}=0.979313 k_{B}\right)$ than the F-P condition, compared to the ALAS envelope (calculated for $\left.k=k_{B}\right)$. the framework of the exact linear wave theory. Note that the simulation duration to reach a steady state for this value of $\delta=0.4$ needs to be significantly increased: $10000 T$ was used for this run.

\section{CONCLUSIONS}

Recently, Couston et al. [4] investigated the applicability of the F-P resonance mechanism to water waves by using two patches of corrugations on an otherwise flat bottom. For small bottom corrugation amplitude and linear waves, they built an asymptotic linear approximate system and solved it by adopting the multiple-scale method, obtaining at leading order an asymptotic linear analytical solution (ALAS). The ALAS is the envelope of the wave amplitude as a function of the space coordinate $x$. The reflection coefficient, transmission coefficient, and enhancement factor are also derived analytically for the F-P resonance. In the linear framework, the enhancement factor increases exponentially as the relative amplitude of the bottom corrugations increases.

The influence of the assumption of small corrugation amplitude adopted in the ALAS derivation was studied here by using an accurate numerical model solving the linear water wave problem with either the exact BBC (system A) or the first-order approximate BBC (system B). A detailed study was performed for the particular case where the ratio of corrugation amplitude to water depth $\delta=d / h$ varies in the range $[0.05,0.4]$, using the same settings as in Ref. [4] for the other geometrical parameters. When the corrugation amplitude is small, for example $\delta=0.05$, predictions from the ALAS are in good agreement with the numerical simulations. For larger values, however, we observe that the resonance does not manifest under the F-P resonance condition. Based on further analyses of the cases with finite corrugation amplitudes $\delta=$ 0.2 and 0.4 , the following conclusions can be drawn.

(1) If the finite amplitude of bottom corrugations is taken into account, F-P resonance does not occur for incident waves with a wave number equal to the target resonant wave number $k_{B}=k_{b} / 2$. No matter whether the exact $\mathrm{BBC}$ or the approximate $\mathrm{BBC}$ is considered, the simulation results show that waves are almost fully reflected by the finite-amplitude bottom corrugations ( $R^{\mathrm{FP}} \rightarrow 1$ before the first patch) and that standing waves are still formed in the resonator but the enhancement factors are not comparable to the values predicted by the ALAS. For example, for the case $\delta=0.2$, in the ALAS, the reflection coefficient $R_{\mathrm{ALAS}}^{\mathrm{FP}} \approx 0.462$ and the enhancement factor $E_{\mathrm{ALAS}}^{\mathrm{FP}} \approx 5.786$, however, $R_{A}^{\mathrm{FP}} \approx 0.956$ and $E_{A}^{\mathrm{FP}} \approx 1.711$ are observed in the simulation with system A. Clearly, F-P resonance is not realized in the simulations of system A or system B at the expected wave number $k_{B}$ when $\delta>0.1$.

(2) By slightly decreasing the wave number of incident waves, however, a situation close to the predicted F-P resonance can be reached with systems A and B. Even for the case with the highest corrugation amplitude studied in the present work, $\delta=0.4$, the resonance can be recovered for the incident wave number $k=0.979313 k_{B}$. In this case, the reflection coefficient and enhancement factor from the numerical simulation with system $\mathrm{A}$ are $R_{A}^{\mathrm{FP}} \approx 0.687$ and $E_{A}^{\mathrm{FP}} \approx 26.918$, respectively. As shown in Fig. 12, the agreement between the 
ALAS prediction and the simulation result is good, with a relative error of enhancement factor of only $2 \%$ compared to the ALAS value, $E_{\mathrm{ALAS}}^{\mathrm{FP}} \approx 27.579$. This demonstrates that F-P resonance can be effectively realized for a finite corrugation amplitude within the linear wave theory, but only for specifically detuned incident wave numbers.

(3) In line with the previous conclusion, it appears that the range of incident wave numbers prone to F-P resonance is extremely narrow for high corrugation amplitudes. As illustrated in Fig. 9 for $\delta=0.2$ and Fig. 11 for $\delta=0.4$, as soon as the incident wave number departs from the optimal value, even slightly, the resonance can no longer develop. The incident waves are then strongly reflected by the two-patch system. The deviation of the optimal wave number from the F-P resonance condition $k=k_{B}$ increases with the amplitude of bottom corrugations. This extreme narrowness of the resonance range makes the possibility of realizing such resonance for practical coastal applications questionable, or, at least, attainable for only very particular incident wave conditions, all the more so as the required duration to reach maximum amplification of waves in the resonator area was observed to increase roughly exponentially as a function of $\delta$.

(4) The fact that the resonant wave number cannot be accurately predicted by the ALAS for finite-amplitude bottom corrugations is mainly related to the implied assumption of the ALAS that the wave number maintains a constant value over the patch of corrugations. In fact, when waves propagate over a patch of corrugations, the effective wave number is slightly higher over this area, as highlighted by the analysis in [13] based on a third-order expansion of the linear dispersion relation. In the numerical model used here, which can cope with arbitrary bottom shapes, the variations of local wave properties due to a variable bottom are fully accounted for, even in the linear framework. This explains the difference between the numerical model results and the ALAS for prediction of the wave-number downshift. Equation (18) can be used to provide a primary estimate of the wave-number downshift.

(5) On the other hand, the approximation related to the first-order expansion of the BBC (i.e., considering system B instead of system A) appears to have less influence. At least for the range of bottom corrugation amplitudes $\delta \in[0.05,0.2]$ considered in the present study, the differences in the results for system A vs system B are quite limited.

In the near-future, the effects associated with the assumption of small-amplitude surface waves will be studied by using the fully nonlinear wave model, (14), and applying the fully nonlinear version of WHISPERS-3D. Finite-amplitude incident waves will introduce higher-order modes due to wave-bottom interactions. Furthermore, the dispersion relation will be affected by the finite amplitude of waves, which will certainly influence the occurrence of F-P resonance, as we have shown in this work that the F-P condition is very sensitive to the effective wave number over the patches of corrugations.

\section{ACKNOWLEDGMENTS}

The authors wish to thank Louis-Alexandre Couston for several discussions on various aspects of Bragg and FabryPerot resonance and suggestions on a preliminary version of this article. J.Z. gratefully acknowledges the financial support from the China Scholarship Council covering his Ph.D. thesis (Grant No. 201604490045).
[1] C. Fabry and A. Pérot, Ann. Chim. Phys. 12, 459 (1897).

[2] J. M. Vaughan, The Fabry Perot Interferometer: History, Theory, Practice and Applications (Taylor \& Francis Group, New York, 1989).

[3] N. Ismail, C. C. Kores, D. Geskus, and M. Pollnau, Opt. Express 24, 16366 (2016).

[4] L. A. Couston, Q. C. Guo, M. Chamanzar, and M. R. Alam, Phys. Rev. E 92, 043015 (2015).

[5] A. G. Davies, J. Mar. Res. 40, 331 (1982).

[6] A. G. Davies and A. D. Heathershaw, J. Fluid Mech. 144, 419 (1984).

[7] A. D. Heathershaw, Nature 296, 343 (1982).

[8] C. C. Mei, J. Fluid Mech. 152, 315 (1985).

[9] J. Yu and C. C. Mei, J. Fluid Mech. 404, 251 (2000).

[10] V. Rey, É. Guazzelli, and C. C. Mei, Phys. Fluids 8, 1525 (1996).

[11] É. Guazzelli, V. Rey, and M. Belzons, J. Fluid Mech. 245, 301 (1992).

[12] L. A. Couston, M. A. Jalali, and M. R. Alam, J. Fluid Mech. 815, 481 (2017).

[13] Y. M. Liu and D. K. P. Yue, J. Fluid Mech. 356, 297 (1998).

[14] T. Hara and C. C. Mei, J. Fluid Mech. 178, 221 (1987).
[15] L. N. Howard and J. Yu, J. Fluid Mech. 593, 209 (2007).

[16] J. Yu and L. N. Howard, J. Fluid Mech. 659, 484 (2010).

[17] J. Yu and G. F. Zheng, J. Fluid Mech. 713, 362 (2012).

[18] P. D. Weidman, A. Herczynski, J. Yu, and L. N. Howard, J. Fluid Mech. 777, 122 (2015).

[19] R. A. Dalrymple and J. T. Kirby, J. Waterway, Port, Coastal, Ocean Eng. 112, 309 (1986).

[20] J. T. Kirby, J. Fluid Mech. 162, 171 (1986).

[21] R. Porter and D. Porter, J. Fluid Mech. 434, 301 (2001).

[22] D. G. Dommermuth and D. K. P. Yue, J. Fluid Mech. 184, 267 (1987).

[23] V. E. Zakharov, J. Appl. Mech. Tech. Phys. 9, 190 (1968).

[24] M. L. Yates and M. Benoit, Int. J. Numer. Methods Fluids 77, 616 (2015).

[25] C. Raoult, M. Benoit, and M. L. Yates, Coastal Eng. 114, 194 (2016).

[26] Y. Tian and S. Sato, Coastal Eng. J. 50, 369 (2008).

[27] M. Benoit, C. Raoult, and M. L. Yates, Wave Motion 74, 159 (2017).

[28] P. A. Madsen, D. R. Fuhrman, and B. L. Wang, Coastal Eng. 53, 487 (2006).

[29] Y. Goda and Y. Suzuki, in 15th International Conference on Coastal Engineering (Honolulu, HI, 1976), pp. 828-845. 